Bull. Austral. Math. Soc.

VOL. 45 (1992) [395-398]

\title{
ON IMPROPER INTEGRALS OF PRODUCTS OF LOGARITHMIC, POWER AND BESSEL FUNCTIONS
}

\author{
M. Aslam Chaudhry and M. Ahmad
}

In this paper we have evaluated the integral $\int_{0}^{\infty} x^{\nu+1} J_{\nu}(a x) K_{0}(b x) \ln (x / 2 b) d x$. A new integral representation of the Euler constant is shown. Some special cases of the result are discussed and an open problem is posed.

\section{INTRODUCTION}

Integrals of products of Bessel functions and powers have been a matter of curiosity for a long time [1, 2, 7]. Askey, Koornwinder and Rahman [1] have evaluated integrals involving powers and product of Bessel functions. A little attempt has been made to evaluate integrals involving powers, logarithmic and Bessel functions $[4,5]$. These integrals are of extreme importance in many branches of mathematical physics, elasticity, potential theory and applied probability $[6,7]$. In this paper we have evaluated the integral

$$
\int_{0}^{\infty} x^{\nu+1} J_{\nu}(a x) K_{0}(b x) \ln (x / 2 b) d x
$$

We have obtained a new integral representation of the Euler constant

$$
\gamma=-\int_{0}^{\infty} J_{0}(\sqrt{t}) K_{0}(\sqrt{t}) \ln (\sqrt{t}) d t
$$

as a special case of our result. Some other special cases are also discussed and an open problem is posed.

TheOREM. For $a>0, b>0$, and $\nu>-1$,

$$
\int_{0}^{\infty} x^{\nu+1} J_{\nu}(a x) K_{0}(b x) \ln (x / 2 b) d x=(2 a)^{\nu} \frac{\Gamma(\nu+1)}{\left(a^{2}+b^{2}\right)^{\nu+1}}\left[\psi(\nu+1)-\ln \left(a^{2}+b^{2}\right)\right] .
$$

Received 9th May, 1991

The authors are indebted to King Fahd University of Petroleum and Minerals for the excellent research facilities.

Copyright Clearance Centre, Inc. Serial-fee code: 0004-9729/92 \$A2.00+0.00. 
Proof: It is known [5, p.340] that

$$
\int_{0}^{\infty} x^{\alpha-1} \exp \left(-s x-b x^{-1}\right) d x=2(b / s)^{\alpha / 2} K_{\alpha}(2 \sqrt{b s}), \quad a>0, s>0 .
$$

A formal differentiation with respect to $\alpha$ yields

$$
\begin{aligned}
\int_{0}^{\infty} x^{\alpha-1}(\ln x) \exp (-s x & \left.-b x^{-1}\right) d x \\
= & 2(b / s)^{\alpha / 2}\left\{\frac{1}{2} \ln (b / s) K_{\alpha}(2 \sqrt{b s})+\frac{\partial}{\partial \alpha} K_{\alpha}(2 \sqrt{b s})\right\} .
\end{aligned}
$$

The process of differentiation in (4) is justified [3, p.430].

Substituting $\alpha=0$ in (4) and using the fact that $(\partial / \partial \alpha)\left[K_{\alpha}(z)\right]_{\alpha=0}=0[5, p .358]$ we get

$$
\int_{0}^{\infty} \frac{\ln x}{x} \exp \left(-s x-b x^{-1}\right) d x=\ln (b / s) K_{0}(2 \sqrt{b s}), \quad b>0, s>0 .
$$

We can write equation (5) in the operational form as follows

$$
L\left\{\frac{\ln x}{x} \exp (-b / x) ; s\right\}=\ln (b / s) K_{0}(2 \sqrt{b s})
$$

where $L$ is the Laplace transform operator [4, p.129].

However, [4, p.132]

$$
L\left\{x^{\nu-1} f(1 / x) ; s\right\}=s^{-\nu / 2} \int_{0}^{\infty} x^{\nu / 2} J_{\nu}(2 \sqrt{x s}) F(x) d x
$$

where $F(s)$ is the Laplace transform of $f(x)$. It follows from (6) and (7) that

(8) $\int_{0}^{\infty} x^{\nu / 2} J_{\nu}(2 \sqrt{s x}) K_{0}(2 \sqrt{b x}) \ln (x / b) d x=s^{\nu / 2} \int_{0}^{\infty} x^{\nu}(\ln x) \exp (-(b+s) x) d x$

However [5, p.576]

$$
\int_{0}^{\infty} x^{\nu-1}(\ln x) e^{-\mu x} d x=\frac{\Gamma(\nu)}{\mu^{\nu}}[\psi(\nu)-\ln \mu], \quad \mu>0, \nu>0
$$

Therefore, it follows from (8) and (9) that

$$
\begin{gathered}
\int_{0}^{\infty} x^{\nu / 2} J_{\nu}(2 \sqrt{s x}) K_{0}(2 \sqrt{b x}) \ln (x / b) d x=s^{\nu / 2} \frac{\Gamma(\nu+1)}{(b+s)^{\nu+1}}[\psi(\nu+1)-\ln (b+s)] . \\
(b>0, s>0, \nu>-1) .
\end{gathered}
$$


Now replacing $s$ by $a^{2}, b$ by $b^{2}$ and then by using the transformation $2 \sqrt{x}=t$, we get the proof of the theorem.

\section{Corollary 1.}

$$
\int_{0}^{\infty} J_{0}(2 \sqrt{a x}) K_{0}(2 \sqrt{b x}) \ln (x / b) d x=\frac{-1}{(a+b)}(\gamma+\ln (a+b)), \quad a>0, b>0
$$

where $\gamma$ is the Euler constant [5].

Proof: This follows from (10) when we take $\nu=0$ and $s=a$.

Corollary 2 .

$$
\gamma=-\int_{0}^{\infty} J_{0}(\sqrt{t}) K_{0}(\sqrt{t}) \ln (\sqrt{t}) d t
$$

Proof: This follows from (11) when we take $a=b=1 / 2$ and use the transformation $2 x=t$.

\section{Corollary 3 .}

$$
\int_{0}^{\infty} \sin (2 \sqrt{a x}) K_{0}(2 \sqrt{b x}) \ln (x / b) d x=\frac{\pi \sqrt{a}}{(a+b)^{3 / 2}}[\psi(3 / 2)-\ln (a+b)], \quad a>0, b>0
$$

Proof: This follows from (10) when we take $\nu=1 / 2$ and use the fact that [5, p.966]

$$
J_{1 / 2}(z)=\sqrt{\frac{2}{\pi z}} \sin z .
$$

In particular, when $a=b=1 / 2$ in (13) we get

$$
\begin{aligned}
& \int_{0}^{\infty} \sin (\sqrt{2 x}) K_{0}(\sqrt{2 x}) \ln (\sqrt{2 x}) d x=\frac{\pi}{2 \sqrt{2}}[2-2 \ln 2-\gamma]=0.6816-1.1107 \gamma \\
& \text { or } \quad \int_{0}^{\infty} x \sin (x) K_{0}(x) \ln (x) d x=\frac{\pi}{4 \sqrt{2}}[2-2 \ln 2-\gamma]=0.3408-0.5554 \gamma .
\end{aligned}
$$

\section{Corollary 4.}

$$
\int_{0}^{\infty} \cos (2 \sqrt{a x}) K_{0}(2 \sqrt{b x}) \ln (x / b) \frac{d x}{\sqrt{x}}=\frac{\pi}{\sqrt{a+b}}[\psi(1 / 2)-\ln (a+b)] .
$$

Proof: This follows from (10) when we taken $\nu=-1 / 2$ and use the fact [5, p.966]

$$
J_{-1 / 2}(z)=\sqrt{\frac{2}{\pi z}} \cos z
$$


In particular when $a=b=1 / 2$ in (14) we get

or

$$
\begin{gathered}
\int_{0}^{\infty} \cos (\sqrt{2 x}) K_{0}(\sqrt{2 x}) \ln (\sqrt{2 x}) \frac{d x}{\sqrt{x}}=-\frac{\pi}{2}(\gamma+2 \ln 2) \\
\int_{0}^{\infty} \cos (t) K_{0}(t) \ln (t) d t=-\frac{\pi}{2 \sqrt{2}}(\gamma+2 \ln 2) .
\end{gathered}
$$

Corollary 5. See $[5$, p.577].

$$
\int_{0}^{\infty} \frac{\ln x}{x} \exp (-\mu(x / c+c / x)) d x=2 \ln c K_{0}(2 \mu), \quad c>0, \mu>0 .
$$

Proof: This follows from (5) when we take $s=\mu / c$ and $b=\mu c$.

For some suitable values of the constants $\rho, a, b$ and $c$ evaluate the integral. The following problem remains open

$$
\int_{0}^{\infty} x^{\rho}(\ln (a x))^{n} J_{\alpha}(b x) K_{\beta}(c x) d x, \quad n \geqslant 2 .
$$

\section{REFERENCES}

[1] R. Askey, T.H. Koornwinder and M. Rahman, 'An integral of products of ultraspherical functions and a q-extension', J. London Math. Soc. 33, 133-148.

[2] W.N. Bailey, 'Some infinite integrals involving Bessel functions', Proc. London math. Soc. 40 (1936), 37-48.

[3] B.M. Budak, S.V. Fomin, Multiple integrals, field theory and series (Mir Publishers, 1978).

[4] A. Erdelyi, W. Magnus, F. Oberhettinger and F.G. Tricomi, Integral transforms, Vol. I (McGRaw-Hill, 1954).

[5] I.S. Gradshteyn and I.M. Ryzhik, Tables of integrals, series and products (Academic Press, 1980).

[6] N.N. Lebedev, Special functions and their applications (Dover Publications Inc., 1972).

[7] G.N. Watson, Theory of Bessel functions, 2nd edition (Cambridge University Press, 1944).

Department of Mathematical Sciences

King Fahd University of Petroleum and Minerals

Dhahran

Saudi Arabia 